\title{
A METHOD OF SOLUTION OF FIELD PROBLEMS BY MEANS OF OVERLAPPING REGIONS*
}

\author{
BY \\ H. PORITSKY AND M. H. BLEWETT \\ General Electric Company
}

1. Introduction. In problems involving the determination of fields, it often happens that the region $R$ for which the field is to be determined is difficult to handle directly, but can be broken up into several overlapping regions $R_{1}, R_{2}, \cdots$ for each of which the field can be determined by standard methods. We suppose that the breaking up is carried out in such a manner that every point of the region $R$ falls into at least one of the regions $R_{1}, R_{2}, \cdots$. In the following, Schwartz' "alternating procedure" is applied to the solution of field problems for such regions $R$.

To illustrate, let us consider the determination of a function $u$ harmonic over the region $R$ shown in Fig. 1, bounded by two circular arcs $A B C$ and $C D A$ with centers at $O$ and $O^{\prime}$. For simplicity we assume that the radii of the two circles are equal and the boundary values of $u$ are symmetric about the straight line through $A$ and $C$. It will be noticed that by completing the circular arcs by means of the dotted curves $A E C$ and $C F A$ one obtains the circular regions over which the determination of a harmonic function in terms of boundary values is well known. Here $R$ is the region

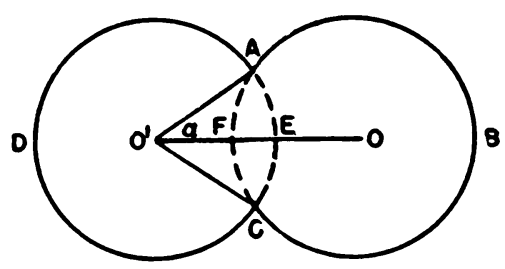

Fig. 1. bounded by the solid circular arcs $A B C$ and $C D A$, while the regions $R_{1}$ and $R_{2}$ are the circular regions bounded by the complete circles with centers at $O$ and $O^{\prime}$. The problem then is to utilize the relatively easy solution of the Dirichlet problem for the circular regions $R_{1}$ and $R_{2}$ in an efficient manner toward the solution of the Dirichlet problem over $R$.

This is done by assuming the values of $u$ over the arc $A F C$; the solution of the Dirichlet problem for the circle $R_{1}$ with center $O$, based on these assumed values and on the known boundary values over $A B C$, is then utilized to furnish the values of $u$ over $A E C$. The procedure is then repeated by solving the Dirichlet problem for the circle $R_{\mathbf{2}}$ with center at $O^{\prime}$, and the values over $A F C$ are recalculated. By alternating between $R$ and $R^{\prime}$ in this way, continual improvement of the values of $u$ over both arcs $A F C$ and $A E C$ results; in the limit this leads to a solution of the Dirichlet problem for the region of Fig. 1.

In the following we shall illustrate the procedure, not for the Laplace equation

but for the equation

$$
\nabla^{2} u=0,
$$

$$
\left(\nabla^{2}+k^{2}\right) u=0
$$

which is encountered in wave motion under the assumption of sinusoidal oscillations, for the region shown in Fig. 2. Other cases of interest in connection with (1.2) which

\footnotetext{
* Received June 8, 1945.
} 
can also be treated by the present method are given by the "open end correction of an organ pipe," wave passage through a change of width of a channel, $T$-sections, etc.

2. Wave propagation around a corner. We consider a solution of the differential equation (1.2) for the region shown in Fig. 2; this solution is to satisfy the boundary conditions

$$
\begin{aligned}
& \frac{\partial u}{\partial n}=0 \text { on } D O G, E B F, \\
& u=A_{1} e^{i k x}+B_{1} e^{-i k x} \text { for large positive } x, \\
& u=A_{3} e^{i k y}+B_{3} e^{-i k y} \text { for large positive } y,
\end{aligned}
$$

where $A_{1}, B_{1}, A_{3}$ and $B_{3}$ are proper constants. Equations (2.2) and (2.3) can be described physically by the statement that $u$ behaves as a plane wave at infinity.

The above problem is encountered in the propagation of a transverse electromagnetic wave around a corner or through a channel the section of which is shown in Fig. 2. Here the channel has an infinite depth in the $z$-direction; the field components are assumed to be independent of $z$, and the only non-vanishing magnetic field component is $H_{z}$. At the boundaries, which are assumed to be metallic and perfectly conducting, the electric field is normal; this leads to the vanishing of the normal derivative

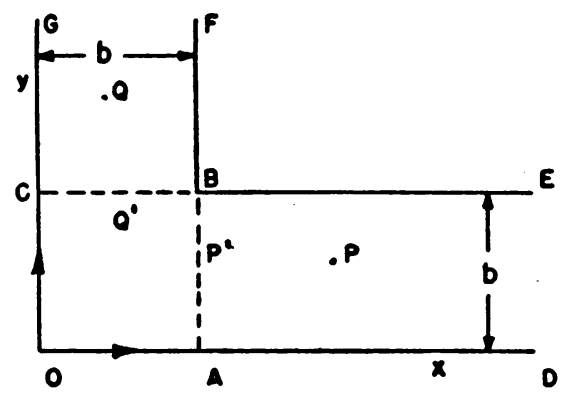

Fig. 2. of $H_{z}$, i.e., $\partial H_{z} / \partial n=0$. Formulation of the field in terms of $H_{z}$ leads to the above problem.

On account of the vanishing of the normal derivative over the $y$-axis, reflection

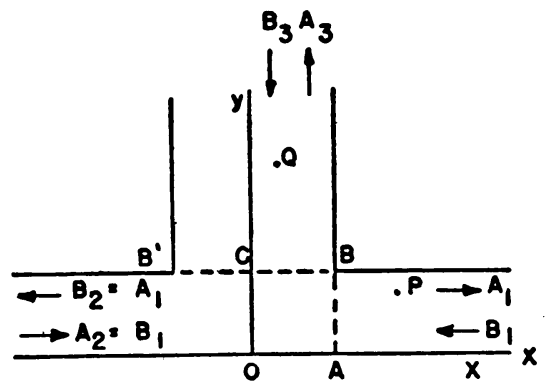

FIG. 3. across it is possible, thus extending the region of Fig. 2 into the region shown in Fig. 3. This reflection is carried out in accordance with the relation

$$
u(-x, y)=u(x, y) \text {. }
$$

In view of this reflection the behavior of $u$ at $x=-\infty$ is given by the expression

$$
u=B_{1} e^{i k x}+A_{1} e^{-i k x} \text {. }
$$

As a result of this reflection the semi-infinite strip DOCE of Fig. 2 can be replaced by the 2-way infinite strip of Fig. 3

$$
-\infty<x<\infty, \quad 0<y<b \text {. }
$$

Similar reflection can be carried out across the lower boundary $y=0$ of Fig. 3 ; this allows us to replace the semi-infinite vertical channel by a vertical channel extending to infinity both up and down. A proper behavior for $u$ at $y=-\infty$ can be obtained from (2.3). 
The general procedure which was outlined in $\$ 1$ is applied to the present case. First, we consider the strip $0<y<b$ of Fig. 3, and assume values for $\partial u / \partial n$ over the dotted part $B^{\prime} C B$ of its upper boundary. Since $\partial u / \partial n$ vanishes over the rest of its boundary and the behavior of $u$ at $\infty$ is described by (2.2) and (2.5), it is possible to determine $u$ at any point interior to this strip. This determination is carried out by means of a Green's function $G$. The derivation of $G$ will be described presently; for the present it will suffice to say that the value of $u$ at an interior point $P$ of the strip is given by the relation

$$
u_{p}=u\left(x_{0}, y_{0}\right)=2 B_{1} \cos k x_{0}+\frac{1}{2 \pi} \int_{-b}^{b}\left(\frac{\partial u}{\partial y}\right)_{y=b} G d x .
$$

$G$ has a pole at $P=\left(x_{0}, y_{0}\right)$, and (2.6) requires that $G$ be evaluated on the dotted line $B^{\prime} C B$. After $u$ is determined in this way, differentiation of (2.6) with respect to $x$ allows one to compute $\partial u / \partial x$, and in particular to determine this derivative over $A B$. Turning now to the infinite vertical strip $0<x<b$, we repeat the same procedure and determine the function $u$ at any point interior to this strip; in particular, we evaluate $u$ and $\partial u / \partial y$ over $C B$. The process is then repeated.

The definition of the Green's function for the differential equation (1.2) and the boundary condition (2.1) for a finite region $R$ is specified by the following:

a) $G$ satisfies (1.2) everywhere in $R$ except at the pole $P$;

b) $\partial G / \partial n$ vanishes along the boundary of $R$;

c) at the pole $P, G$ becomes infinite like $-\ln r^{\prime}$, where $r^{\prime}$ is the distance from $P$.

We apply Green's theorem in the form

$$
\int \cdot\left[u\left(\nabla^{2}+k^{2}\right) v-v\left(\nabla^{2}+k^{2}\right) u\right] d A=\int\left(u \frac{\partial v}{\partial n}-v \frac{\partial u}{\partial n}\right) d s
$$

to the region $R$, exclude the neighborhood of the point by means of a small circle of radius $\epsilon$ and let $\epsilon$ approach zero. This yields the equation

$$
u_{p}=\frac{1}{2 \pi} \int \frac{\partial u}{\partial n} G d s,
$$

where the integration is carried out over the boundary of $R$. In the present case, for the infinite strip $0<y<b$ special additional considerations are required. It will be assumed that in addition to the requirements (2.7) the Green's function $G$ behaves at infinity like a divergent plane wave. Solutions of (1.2) which depend on $x$ only are

$$
e^{ \pm i k x}
$$

We consider the wave equation

$$
\frac{\partial^{2} U}{\partial t^{2}}=c^{2} \nabla^{2} U
$$

and look for solutions of the form $u e^{ \pm i \omega t}$. If we set $k=\omega / c$, we find that $u$ satisfies Eq. (1.2), and that $e^{i k x}$ represents a plane wave traveling in the direction of positive $x$ while $e^{-i k x}$ represents a similar wave traveling in the direction of negative $x$. It will 
be assumed that at $x= \pm \infty$ the Green's function $G$ behaves like a divergent plane wave of the same amplitude at $x=+\infty$ as at $x=-\infty$.

It will be assumed that the dimension $b$ satisfies the inequality

$$
b<\pi / k .
$$

Physically this assumption means that the width $b$ of the strip is less than half the wave length $\lambda / 2=\pi / k$ of a plane wave at the frequency in question. The effect of this assumption and the features which arise when it is not satisfied will appear presently.

First, we place the pole $P$ on the $y$-axis. We shall obtain $G$ as a series in the form

$$
G=\sum_{n=0}^{\infty} A_{n} u_{n}
$$

where $u_{n}$ are product solutions of the wave equation (1.2), i.e., $u_{n}$ consist of the product of a function of $x$ and a function of $y$; more explicitly,

$$
\left.\begin{array}{l}
u_{0}=\exp [i k \cdot|x|], \\
u_{n}=\cos \frac{n y}{b} \exp \left[-\sqrt{\left(\frac{n \pi}{b}\right)^{2}-k^{2} \cdot|x|}\right], \quad(n>0) .
\end{array}\right\}
$$

These product solutions $u_{n}(n>0)$ have been chosen so that they don't become infinite at $x= \pm \infty$, while $u_{0}$ represents a divergent plane wave. If the inequality (2.12) were not satisfied, several radicals in $u_{n}(n>0)$ would be imaginary, infinitely large values of $u_{n}$ could not be avoided, and additional stipulations regarding the behavior of $G$ at infinity would have to be made.

The functions $u_{n}$ are symmetric about the vertical line $x=0$ through the pole $P$, and continuous at $x=0$. However, $\partial u_{n} / \partial x$ is discontinuous at $x=0$, the discontinuity being

$$
\Delta\left(\frac{\partial u_{n}}{\partial x}\right)=\left\{\begin{array}{lll}
-2 i k & \text { for } & n=0 \\
2 \sqrt{\left(\frac{n \pi}{k}\right)^{2}-k^{2} \cos \frac{n \pi y}{b}} & \text { for } & n>0 .
\end{array}\right\}
$$

Thus each term $u_{n}$ may be considered as representing the wave function corresponding to a sinusoidal distribution of sources* over the line $x=0$. The density $\sigma$ of the sources is given by the familiar condition from potential theory

$$
\text { discontinuity in normal derivative }=\Delta\left(\frac{\partial u}{\partial x}\right)=-2 \pi \sigma,
$$

and in the present case is given by

$$
\sigma_{n}=\frac{1}{\pi} \sqrt{\frac{n^{2} \pi^{2}}{b^{2}}-k^{2} \cos \frac{n \pi y}{b}} .
$$

* By a "source" is meant here a solution of (1.1) which depends only upon the distance $r$ from a fixed point, is singular at $r=0$ like $-\ln r$, and behaves at infinity like a divergent cylindrical wave. The distributions of such sources satisfy continuity-discontinuity relations similar to those in the case of logarithmic potentials. 
For (2.13) this yields

$$
\sigma=\frac{1}{\pi} \sum_{n=0}^{\infty} A_{n} \sqrt{\frac{n^{2} \pi^{2}}{b^{2}}-k^{2} \cos \frac{n \pi y}{b}} .
$$

Let us now consider the concentrated point source at the pole $P$, and express it as a Fourier series of cosines over the interval $x=0,0<y<b$, obtaining

$$
\sigma=\frac{1}{b}+\frac{2}{b} \sum_{n=1}^{\infty} \cos \frac{n \pi y_{0}}{b} \cos \frac{n \pi y}{b}
$$

where $x=0, y=y_{0}$ are the coordinates of the pole $P$. Solving for $A_{n}$, we obtain for the Green's function $G$ the Fourier series

$$
\begin{aligned}
G=\frac{2 \pi}{b}\{ & -\frac{1}{2 i k} \exp [i k \cdot|x|] \\
& \left.+\sum_{n=1}^{\infty} \frac{\cos \left(n \pi y_{0} / b\right)}{\sqrt{(n \pi / b)^{2}-k^{2}}} \cos \frac{n \pi y}{b} \exp \left[-\sqrt{\left(\frac{n \pi}{b}\right)^{2}-k^{2} \cdot|x|}\right]\right\} .
\end{aligned}
$$

Due to the behavior of $G$ at infinity it is found that after applying the Green's theorem (2.8) over the rectangular region $-l^{\prime}<x<l$ and letting $l$ and $l^{\prime}$ recede to infinity, certain additional terms $R^{\prime}$ and $R^{\prime \prime}$ arise from the boundaries $x=l$ and $x=l^{\prime}$. Equation (2.9) is now replaced by

$$
u\left(x_{0}, y_{0}\right)=\left.\frac{1}{2 \pi} \int_{-l^{\prime}}^{l} \frac{\partial u(x, y)}{\partial y}\right|_{y=b} G d x+R^{\prime}+R^{\prime \prime}
$$

where

$$
\begin{aligned}
R^{\prime} & =\left.\frac{1}{2 \pi} \int_{0}^{b}\left(\frac{\partial u}{\partial x} G-u \frac{\partial G}{\partial x}\right) d y\right|_{x=l}, \\
R^{\prime \prime} & =\left.\frac{1}{2 \pi} \int_{0}^{b}\left(-\frac{\partial u}{\partial x} G+u \frac{\partial G}{\partial x}\right) d y\right|_{x=-l^{\prime}} .
\end{aligned}
$$

In view of (2.4), (2.2), (2.20), (2.21) and (2.22), Eq. (2.21) can be given the form (2.6).

As explained above, in the present case not (2.6) but its $x$-derivative will be found useful. Differentiation of (2.6) yields

$$
\frac{\partial u\left(x_{0}, y_{0}\right)}{\partial x_{0}}=-2 k B_{1} \sin k x_{0}+\frac{1}{2 \pi} \int_{-b}^{b}\left(\frac{\partial u}{\partial y}\right)_{y=b} \frac{\partial G}{\partial x_{0}} d x .
$$

To obtain this equation, the integral in (2.6) has been differentiated under the integral sign; this is permissable since the limits of integration are independent of $x_{0}$. Since $(\partial u / \partial y)_{y=b}$ is also independent of $x_{0}$, only $G$ has to be differentiated. The explicit form of $(2.24)$ is given by the relation

$$
g\left(y_{0}\right)=\left(\frac{\partial u}{\partial x_{0}}\right)_{x_{0}-b}=-2 k B_{1} \sin k b-\frac{1}{b} \int_{-b}^{b} f(x)\left[K_{0}+\sum_{n=1}^{\infty} K_{n}\right] d x
$$


where

$$
\left.\begin{array}{rl}
f(x) & =\left(\frac{\partial u}{\partial y}\right)_{y=b}, \quad K_{0}=\frac{1}{2} \exp [-i k(x-b)] \\
K_{n} & =(-1)^{n} \cos \frac{n \pi y_{0}}{b} \exp \left[\sqrt{\left(\frac{n \pi}{b}\right)^{2}-k^{2}(x-b)}\right]
\end{array}\right\}
$$

A similar expression holds for $\left(\partial u / \partial y_{0}\right)$ along $C B$;

$$
f\left(x_{0}\right)=\left(\frac{\partial u}{\partial y_{0}}\right)_{y_{0}=b}=-2 k B_{3} \sin k b-\frac{1}{b} \int_{-b}^{b} g(y)\left[K_{0}+\sum K_{n}\right] d y
$$

where $K_{0}, K_{n}$ are as in (2.26) but with the coordinates interchanged.

In applying (2.25) one must assume not only $f(x)$ but also $B_{1}$. Likewise in applying (2.27), $B_{3}$ is required along with $g(y)$. Furthermore, $A_{1}$ and $A_{3}$ are essential to the complete solution. In this connection it is advisable to keep the following relations between $f(x), g(y)$ and the constants $A_{1}, B_{1}, A_{3}$ and $B_{3}$ in mind:

$$
\begin{gathered}
A_{1}=B_{1}-\frac{1}{2 i k b} \int_{-b}^{b} f(x) e^{-i k x} d x, \\
A_{3}=B_{3}-\frac{1}{2 i k b} \int_{-b}^{b} g(y) e^{-i k y} d y, \\
A_{1} e^{i k b}-B_{1} e^{-i k b}=\frac{1}{2 i k b} \int_{-b}^{b} g(y) d y, \\
A_{3} e^{i k b}-B_{3} e^{-i k b}=\frac{1}{2 i k b} \int_{-b}^{b} f(x) d x .
\end{gathered}
$$

These relations enable one to express $A_{1}, B_{1}, A_{3}$ and $B_{3}$ in terms of $f(x)$ and $g(y)$.

The relation (2.28) is established by applying (2.6) to $u\left(x_{0}, y_{0}\right)$ for $x_{0}$ so large that $G$ reduces to its first term in (2 20), and comparing the result with (2.2). A similar derivation over $0<x<b$ yields (2.29). As regards (2.30) it is established by expanding $\partial u / \partial x$ in the horizontal strip $0<y<b$ in a series of cosines of $n \pi y / b$ and comparing for large positive $x$ this expansion with $\partial u / \partial x$ as derived from $(2.2)$; a similar procedure applied over the vertical strip $0<x<b$ to $\partial u / \partial y$ leads to (2.31).

In the present example, in view of the geometric symmetry of the region of Fig. 2 about the diagonal $O B$, any function $u$ over the region can be expressed as the sum of a function which is odd about this diagonal, and one which is even about it. The calculations outlined are simplified considerably for even and odd functions $u$, are quite similar for the two cases and will be illustrated for the odd case.

In the odd case,

$$
\begin{gathered}
A_{3}=-A_{1}, \quad B_{3}=-B_{1}, \\
g(x)=-f(x),
\end{gathered}
$$

and the integral relations $(2.28)-(2.31)$ reduce to

$$
g(y)=-2 k B_{1} \sin k b-\frac{1}{b} \int_{-b}^{b} f(x)\left[K_{0}+\sum_{n=1}^{\infty} K_{n}\right] d x,
$$




$$
\begin{aligned}
A_{1}-B_{1} & =-\frac{1}{2 i k b} \int_{-b}^{b} f(x) e^{-i k x} d x \\
A_{1} e^{i k b}-B_{1} e^{-i k b} & =-\frac{1}{2 i k b} \int_{-b}^{b} f(x) d x
\end{aligned}
$$

After (2.25) has been applied for an initially assumed $f(x)$ curve, the resulting $g(y)$ shape, changed in sign and plotted against $x$, can be considered as the next approximation to $f(x)$, in view of the relation (2.33) and the symmetry of the region about $x=y$.

From (2.35) the coefficients $A_{1}$ and $B_{1}$ may be determined in terms of $(\partial u / \partial y)_{y=b}=f(x)$. This yields

$$
\left.\begin{array}{l}
A_{1}=-\frac{1}{4 k b \sin k b} \int_{-b}^{b} f(x)\left[e^{-i k(x+b)}-1\right] d x, \\
B_{1}=-\frac{1}{4 k b \sin k b} \int_{-b}^{b} f(x)\left[e^{i k b(b-x)}-1\right] d x .
\end{array}\right\}
$$

\begin{tabular}{|c|c|c|c|}
\hline$y_{0}$ & $x$ & $\sum K_{n}$ & $b G / 2 \pi$ \\
\hline $.1 b$ & $\begin{array}{r}.9 b \\
.7 b \\
.5 b \\
.3 b \\
.1 b \\
-.1 b \\
-.3 b \\
-.5 b \\
-.7 b \\
-.9 b\end{array}$ & $\begin{array}{l}-.4347 \\
-.3025 \\
-.1934 \\
-.1151 \\
-.0682 \\
-.0392 \\
-.0222 \\
-.0126 \\
-.0070 \\
-.0040\end{array}$ & $\begin{array}{r}.0613+.0627 i \\
.1625+.1841 i \\
.2111+.2938 i \\
.2034+.3852 i \\
.1448+.4524 i \\
.0545+.4912 i \\
-.0527+.4991 i \\
-.1671+.4775 i \\
-.2756+.4217 i \\
-.3567+.3410 i\end{array}$ \\
\hline $.3 b$ & $\begin{array}{r}.9 b \\
.7 b \\
.5 b \\
.3 b \\
.1 b \\
-.1 b \\
-.3 b \\
-.5 b \\
-.7 b \\
-.9 b\end{array}$ & $\begin{array}{l}-.4143 \\
-.2546 \\
-.1458 \\
-.0806 \\
-.0453 \\
-.0252 \\
-.140 \\
-.0078 \\
-.0044 \\
-.0025\end{array}$ & $\begin{array}{r}.0817+.0627 i \\
.2104+.1841 i \\
.2587+.2938 i \\
.2379+.3852 i \\
.1677+.4524 i \\
.0679+.4912 i \\
-.0445+.4991 i \\
-.1625+.4775 i \\
-.2730+.4217 i \\
-. .3632+.3410 i\end{array}$ \\
\hline $.5 b$ & $\begin{array}{l}.9 b \\
.7 b \\
.5 b \\
.3 b \\
.1 b\end{array}$ & $\begin{array}{l}-.3348 \\
-.1373 \\
-.0437 \\
-.0133 \\
-.0039\end{array}$ & $\begin{array}{l}.1612+.0627 i \\
.3277+.1841 i \\
.3608+.2938 i \\
.3052+.3852 i \\
.2091+.4524 i\end{array}$ \\
\hline
\end{tabular}

The procedure used consisted in assuming $f(x)$, calculating $A_{1}$ and $B_{1}$ from (2.36),

\begin{tabular}{|c|c|c|c|}
\hline$y_{0}$ & $x$ & $\sum K_{n}$ & $b G / 2 \pi$ \\
\hline $.5 b$ & $\begin{array}{l}-.1 b \\
-.3 b \\
-.5 b \\
-.7 b \\
-.9 b\end{array}$ & $\begin{array}{c}-.0011 \\
-.0003 \\
0 \\
0 \\
0\end{array}$ & $\begin{array}{r}.0920+.4912 i \\
-.0308+.4991 i \\
-.1545+.4775 i \\
-.2686+.4217 i \\
-.3607+.3410 i\end{array}$ \\
\hline $.7 b$ & $\begin{array}{r}.9 b \\
.7 b \\
.5 b \\
.3 b \\
.1 b \\
-.1 b \\
-.3 b \\
-.5 b \\
-.7 b \\
-.9 b\end{array}$ & $\begin{array}{r}-.1433 \\
.1255 \\
.1149 \\
.0725 \\
.0429 \\
.0244 \\
.0138 \\
.0078 \\
.0044 \\
.0025\end{array}$ & $\begin{array}{r}.3527+.0627 i \\
.5905+.1841 i \\
.5194+.2938 i \\
.3910+.3852 i \\
.2559+.4524 i \\
.1181+.4912 i \\
-.0167+.4991 i \\
-.1467+.4775 i \\
-.2642+.4127 i \\
-.3582+.3410 i\end{array}$ \\
\hline $.9 b$ & $\begin{array}{r}.9 b \\
.7 b \\
.5 b \\
.3 b \\
.1 b \\
-.1 b \\
-.3 b \\
-.5 b \\
-.7 b \\
-.9 b\end{array}$ & $\begin{array}{r}1.1279 \\
.5691 \\
.2862 \\
.1387 \\
.0744 \\
.0410 \\
.0226 \\
.0126 \\
.0070 \\
.0040\end{array}$ & $\begin{array}{r}1.6239+.0627 i \\
1.0341+.1841 i \\
.6727+.2938 i \\
.4572+.3852 i \\
.2874+.4524 i \\
.1341+.4912 i \\
-.0079+.4991 i \\
-.1419+.4775 i \\
-.2616+.4217 i \\
-.3567+.3410 i\end{array}$ \\
\hline
\end{tabular}
then applying (2.34) to calculate $g(y)$, and using the shape of the latter with the sign

TABIE 1 
changed as the starting point of the next step. To prevent the solution from becoming infinite, at each step $f(x)$ is divided by $A_{1}$, thus yielding the case $A_{1}=1$. In the following numerical work the assumption $b=0.2 \lambda, k b=72^{\circ}$ is made.

Although from physical considerations one would be able to make a reasonably

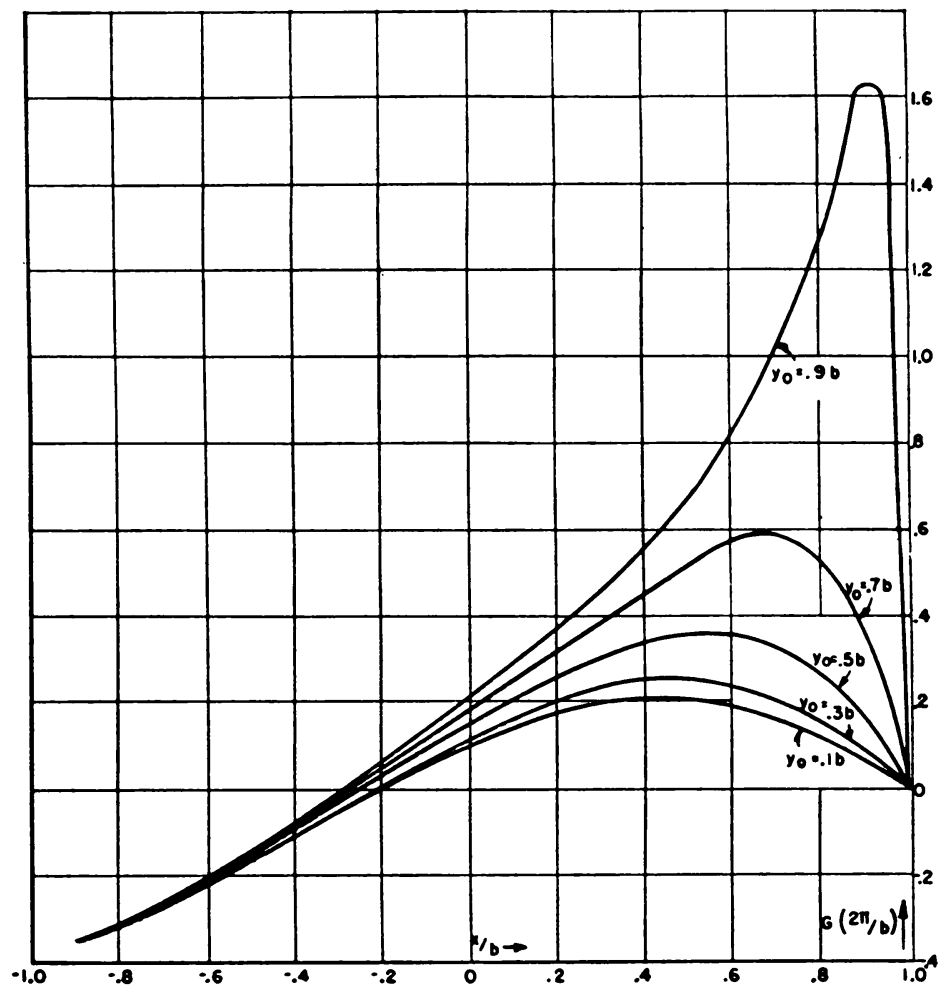

FIG. 4.

good guess for the value of $f(x)$, it was felt that in order to test the method thoroughly, the assumption

$$
\text { along } B C, f(x)=\left(\frac{\partial u}{\partial y}\right)_{y=b}=\text { constant }=1 \text {, }
$$

would be more advisable. Making this assumption, solving for $A_{1}$ and $B_{1}$ from (2.36) (with $b=0.2 \lambda, k b=72^{\circ}$ ), and dividing by $A_{1}$, we obtain

$$
\left.\begin{array}{rl}
A_{1}=1, \quad B_{1} & =\frac{b k-e^{i k b} \sin k b}{b k-e^{-i k b} \sin k b}=.0634-.999 i, \\
f(x) & =\left(\frac{\partial u}{\partial y}\right)_{y=b}=k(1.320-1.238 i) .
\end{array}\right\}
$$

The Green's function was evaluated for five positive and five negative values of $x$, and for five values of $y_{0}$, as shown in Table 1 . The real part of this family of curves is shown in Fig. 4, the imaginary part being merely $(\pi / b) \sin k(x-b)$. These values, with 
(2.38), were inserted in (2.34), the integration being made graphically with areas found by the trapezoidal rule, except near $y=b$.

As $y$ approaches $b$, the value of $g(y)$ increases so rapidly that extrapolation for the curve and the resulting graphical integration is difficult in this region. From physical considerations based on the fact that in a region which is small compared to a wavelength the function $u$ behaves like a harmonic function, it may be shown that a fairly accurate approximation is obtained by assuming $g(y)$ to vary as $(y-b)^{-1 / 3}$ as $y$ approaches $b$. By picking two points $y_{1}$ and $y_{2}$, two constants $A$ and $B$ can be found such that $g(y)=A+B(b-y)^{-1 / 3}$ is fitted to the curve already drawn in this neighborhood for $y<.9 b$; then the area is equal to

$$
\int_{y_{2}}^{b} g(y) d y=A\left(b-y_{2}\right)+\frac{3 B}{2}\left(b-y_{2}\right)^{2 / 3} .
$$

The resulting first approximation for $\partial u / \partial x$ is shown in Table 2. By means of (2.36) the values of $A_{3}$ and $B_{3}$ (the negatives of $A_{1}$

TABLE 2.

\begin{tabular}{c|c}
\hline$y$ & $\begin{array}{l}g(y)=\partial u / \partial y=(\partial u / \partial x \text { for } \\
\text { corresponding values of } x)\end{array}$ \\
\hline $.1 b$ & $-k[1.004-.9445 i]$ \\
$.3 b$ & $-k[1.054-.992 i]$ \\
$.5 b$ & $-k[1.17 \dot{3}-1.103 i]$ \\
$.7 b$ & $-k[1.382-1.299 i]$ \\
$.9 b$ & $-k[1.382-1.299 i]$ \\
$.9 b$ & $-k[1.876-1.761 i]$ \\
\hline
\end{tabular}
and $B_{1}$ ) corresponding to these values were found to be $B_{3}=.1728-1.006 i, A_{3}=.998-.102 i$; thus $B_{1} / A_{1}=.2735-.980 i$.

In order to keep $A_{1}$ fixed at the value unity which we have assumed, we retain this value of the $B_{1} / A_{1}$ ratio and rename it $B_{1}$ as before. We must then divide the values of $\partial u / \partial y$ in Table 2 by $A_{1}$. Reinsertion now into (2.27) gives us the second approximation to $\partial u / \partial y$ shown in Table 3 . The corresponding $A_{1}$ and $B_{1}$ yield the ratio $B_{1} / A_{1}=.2658-.960 i$. The third approximation is then carried out in similar fashion, with the the results shown in Table 4 . In this case, we have $B_{1} / A_{1}=.266-.964 i$. The approximations to $\partial u / \partial y$ are shown in Fig. 5. Figure 6 shows the ratio $B_{1} / A_{1}$ and thus we see that this ratio is converging toward the value $.266-.962 i$, with the absolute value .997 .

TABLE 3.

The second approximation.

\begin{tabular}{c|l}
\hline \hline$x$ & $f(x)=\partial u / \partial y$ \\
\hline $.1 b$ & $k[1.119-.804 i]$ \\
$.3 b$ & $k[1.176-.844 i]$ \\
$.5 b$ & $k[1.309-.960 i]$ \\
$.7 b$ & $k[1.562-1.111 i]$ \\
$.9 b$ & $k[2.100-1.619 i]$ \\
\hline
\end{tabular}

TABLE 4.

The third approximation.

\begin{tabular}{c|c}
\hline \hline$x$ & $f(x)=\partial u / \partial y$ \\
\hline $.1 b$ & $k[1.081-.785 i]$ \\
$.3 b$ & $k[1.132-.821 i]$ \\
$.5 b$ & $k[1.249-.911 i]$ \\
$.7 b$ & $k[1.515-1.095 i]$ \\
$.9 b$ & $k[2.12-1.557 i]$ \\
\hline
\end{tabular}

A similar calculation could be carried out for a function which is even about the diagonal $O B$. The results of this, together with those already found for the odd function, would enable us to cover all cases involving a corner with these dimensions. 


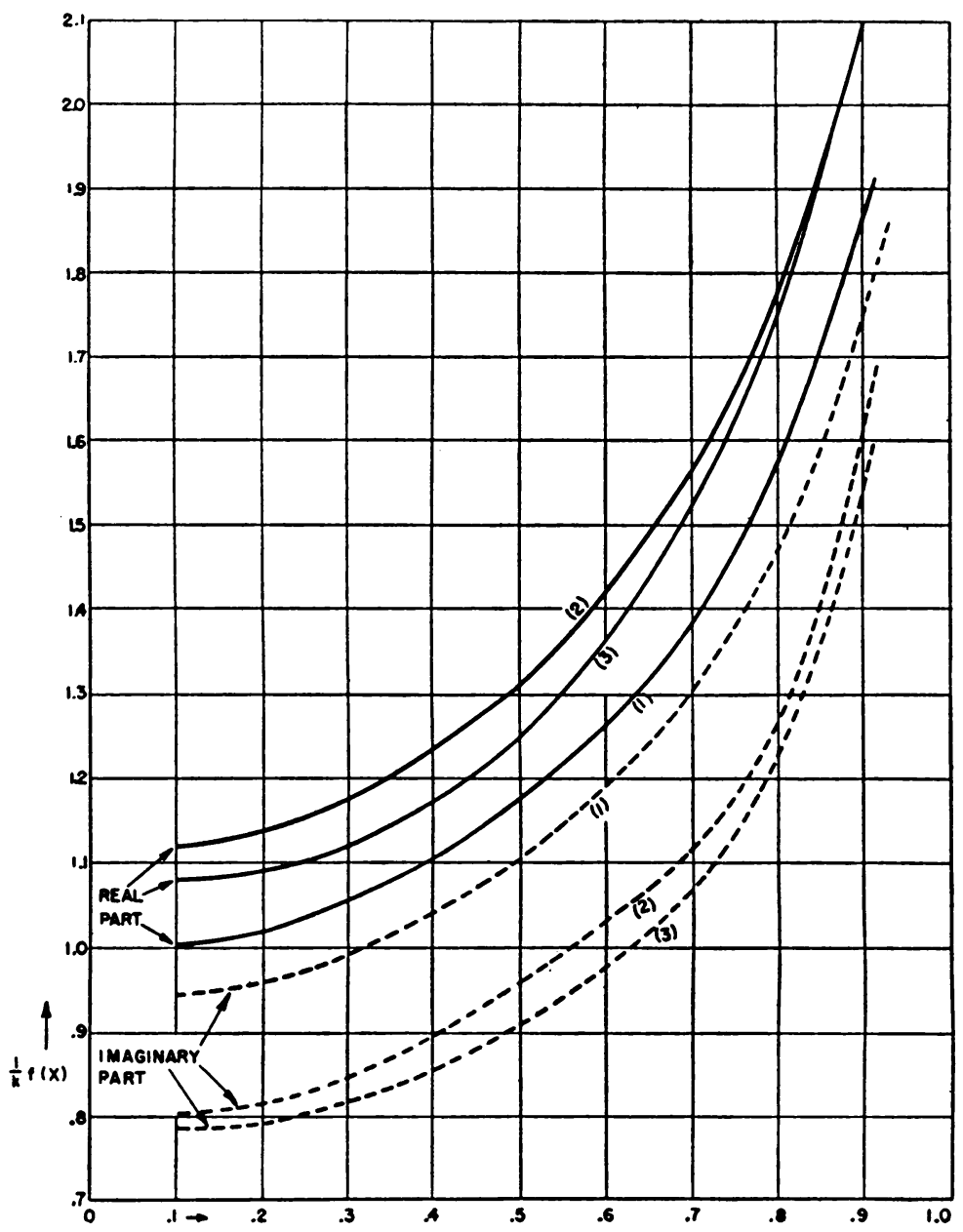

Fig. 5.

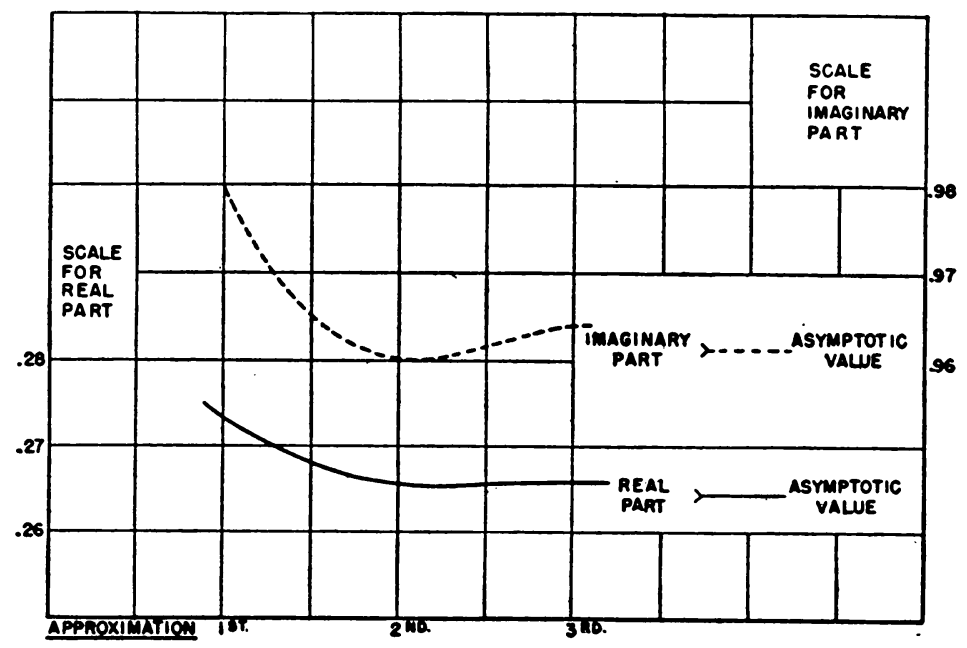

Fig. 6. 
3. An alternative method of procedure. The procedure described and illustrated in the preceding sections can also be applied in a different way. Basically, the calculation was carried out by first assuming the field over the line $C B$ in Fig. 2, then calculating it over the line $B A$. It is possible to carry out the same calculation by assuming the field over $C B$ not as a function of $x$ or as a curve, but as a Fourier cosine series in $x$,

$$
f(x)=\left.\frac{\partial u}{\partial y}\right|_{y=b}=\sum C_{n} \cos \frac{n \pi x}{b} .
$$

Similarly, $g(y)=\partial u / \partial x$ over $A B$ can be converted into a similar Fourier cosine series in $y$,

$$
g(y)=\left.\frac{\partial u}{\partial x}\right|_{x=b}=\sum D_{n} \cos \frac{n \pi y}{b} .
$$

Applying (2.34), (2.35) and (2.26) to the calculation of $g(y)$ from $f(x)$, we obtain

$$
\begin{aligned}
g\left(y_{0}\right) & =\sum D_{m} \cos \frac{m \pi y_{0}}{b} \\
& =-2 k B_{1} \sin k b-\frac{1}{2 b} \sum_{n} C_{n} \int_{-b}^{b} \exp [-i k(x-b)] \cos \frac{n \pi x}{b} d x \\
& -\frac{1}{b} \sum_{m=1}^{\infty} \sum_{n=0}^{\infty}(-1)^{m} C \cos \frac{n \pi y_{0}}{b} \int_{-b}^{b} \cos \frac{n \pi x}{b} \exp \sqrt{\left(\frac{m \pi}{b}-k^{2}(k-b)\right)} d x .
\end{aligned}
$$

This leads to integrals involving a cosine and an exponential in $z$. After these integrations are carried out, each one of the coefficients $D_{n}$ of the expansion (3.2) turns out to be linearly dependent upon the coefficients $C_{n}$. Thus, instead of being given a curve $f(x)$ and computing from it the curve $g(y)$, one starts with $B_{1}$ and a series of coefficients $C_{n}$ represented by the Fourier expansion (3.1) and ends up with the coefficients $D_{n}$ by applying (3.4). The explicit relation between these two sets of coefficients is

$$
D_{0}=-2 k B_{1} \sin k b+\sum_{n=0}^{\infty} P_{0 n} C_{n}, \quad D_{m}=\sum P_{m n} C_{n} \text { for } m>0,
$$

where

$$
\begin{aligned}
& P_{0 n}=\frac{(-1)^{n} i k b\left(1-e^{2 i k b}\right)}{2\left(n^{2} \pi^{2}-k^{2} b^{2}\right)}, \\
& P_{m n}=\frac{(-1)^{n+m+1}\left(m^{2} \pi^{2}-k^{2} b^{2}\right)^{1 / 2}\left\{1-\exp \left\lceil-2\left(m^{2} \pi^{2}-k^{2} b^{2}\right)^{1 / 2}\right]\right\}}{\left(m^{2}+n^{2}\right) \pi^{2}-k^{2} b^{2}}, \quad m>0 .
\end{aligned}
$$

The matrix $P_{m n}$ thus takes the place of the series of curves $\partial G / \partial x$ which were given in Table 1 and shown in Fig. 4. A similar set of equations expresses $C_{n}$ in terms of $D_{n}$ and $B_{3}$.

By proceeding as in $\S 2$ with the field which is odd about the $45^{\circ}$ diagonal $O B$, it is clear that for the final field the coefficients $D_{n}$ should be the negatives of the coefficients $C_{n}$. For the individual successive approximations, this of course is not necessarily the case. The calculation of the next improvement can be carried out by starting with $D_{n}$, changing their signs and putting them in place of $C_{n}$ in (3.4). 
It is possible to replace $D_{m}$ by $C_{m}$ in (3.4). A solution of the resulting equations would lead to a complete determination of the field problem. However, the solution of the resulting equations itself involves some method of successive approximation; hence, this procedure is not advisable, and the successive calculation of $C$ 's and $D$ 's appears to be preferable, since it agrees in spirit with the method outlined above and constitutes just a variation of it. 